PROCEEDINGS OF THE

AMERICAN MATHEMATICAL SOCIETY

Volume 133 , Number 12 , Pages $3447-3453$

S 0002-9939(05)08226-2

Article electronically published on June 28, 2005

\title{
QUASI-E-LOCALLY CYCLIC TORSION-FREE ABELIAN GROUPS
}

\author{
MANFRED DUGAS AND C. J. MAXSON \\ (Communicated by Lance W. Small)
}

\begin{abstract}
For a torsion-free abelian group $A$, we investigate the problem of determining when $\operatorname{End}(A)$ is maximal as a ring in the near-ring of all 0preserving functions on $A$. We introduce the concept of quasi-End(A)-locally cyclic groups and determine several properties of these abelian groups.
\end{abstract}

\section{INTRODUCTION}

Let $A$ be an abelian group and let $M_{0}(A)=\{f: A \rightarrow A: f(0)=0\}$ denote the near-ring of zero-preserving functions on $A$. It is well known that $M_{0}(A)$ is a simple near-ring [7] for any group $A$, not necessarily abelian. On the other hand, $M_{0}(A)$ does contain subrings, for example $\operatorname{End}(A)$, the ring of all endomorphisms of $A$. In [6] the problem of finding the subrings of $M_{0}(A)$ containing $\operatorname{End}(A)$ was initiated. As a result, it was found that every torsion group $A$ has the property that $\operatorname{End}(A)$ is maximal as a ring in $M_{0}(A)$.

To facilitate the investigation mentioned above, the class of $\operatorname{End}(A)$-locally cyclic (E-lc) groups was introduced and it was shown that an E-lc group has the property that $\operatorname{End}(A)$ is a maximal subring of $M_{0}(A)$. Several properties of E-lc groups were found and several classes of E-lc groups were identified. In particular, every torsion group is E-lc.

In this paper we turn our attention to torsion-free groups and later to torsion-free groups of finite rank, i.e. tffr groups. It turns out that for torsion-free groups, a more general condition suffices. We introduce this concept, called quasi-Elc, or qElc for short, and show that whenever $A$ is qElc, then $\operatorname{End}(A)$ is a maximal subring of $M_{0}(A)$. We obtain several properties of qElc groups and give some examples. In particular, we show that the class of qElc groups properly contains the class of all E-lc groups.

In Section 3 we use some work of Arnold [1] to completely characterize the qElc groups of rank 2. In Section 4 we again consider the general case and consider the case of tffr strongly indecomposable groups. We find some sufficient conditions for such groups to be qElc.

Received by the editors July 15, 2004.

2000 Mathematics Subject Classification. Primary 20K30; Secondary 16Y30.

Key words and phrases. Torsion-free groups, endomorphism rings, near-ring of mappings.

This paper was written in part while the second author was visiting the mathematics department of the University of Stellenbosch, South Africa. The gracious hospitality received during this visit is gratefully acknowledged.

(C)2005 American Mathematical Society Reverts to public domain 28 years from publication 3447 
Convention 1. Throughout this paper all groups are abelian and the adjective "abelian" is often omitted. We use $\mathbb{Z}, \mathbb{Z}_{n}, \mathbb{Q}$ to denote the additive group (or ring) of integers, integers modulo $n$, and the rational numbers respectively. Moreover, $\mathbb{N}$ is the set of positive integers. For undefined notation and concepts regarding abelian groups we refer to 3 .

\section{BASIC CONCEPTS}

If $A$ is a torsion-free abelian group, we write $\mathbb{Q} A$ for $\mathbb{Q} \otimes_{\mathbb{Z}} A$. Note that $A$ is an $\operatorname{End}(A)$-module by setting $\varphi \cdot a=\varphi(a)$ for all $\varphi \in \operatorname{End}(A)$ and $a \in A$. The same holds for $\mathbb{Q} A$ and $\mathbb{Q} E n d(A)$. We are now ready for our first definition.

Definition 1. A torsion-free group $A$ is qElc if and only if for all elements $a, b \in A$ there exists some $c \in A$ such that $a, b \in(\mathbb{Q} \operatorname{End}(A)) \cdot c$.

Of course, any E-lc group is qElc. Recall that two torsion-free groups $A, B$ are quasi-equal if there is a non-zero natural number $n$ such that $n A \subseteq B \subseteq A$. Moreover, $A, B$ are quasi-isomorphic if $A, B$ are isomorphic to two quasi-equal groups. We now collect some basic properties of qElc groups, which show that the notion of qElc groups fits nicely into the theory of the category of torsion-free abelian groups with quasi-homomorphisms.

Lemma 1. Let $A, B$ be torsion-free abelian groups. Then the following hold:

(1) If $A$ is quasi-isomorphic to $B$ and $A$ is qElc, then $B$ is qElc.

(2) The following are equivalent:

(2.1) A is qElc.

(2.2) Any finite subset of $A$ is contained in a cyclic $\mathbb{Q} E n d(A)$-submodule of $\mathbb{Q} A$, i.e. $\mathbb{Q} A$ is a locally cyclic $\mathbb{Q}$ End $(A)$-module.

(2.3) For any $a, b \in A$ there are $\alpha, \beta \in \operatorname{End}(A)$ and some $n \in \mathbb{N}, c \in A$ such that $\alpha(c)=n a$ and $\beta(c)=n b$.

(3) If $A$ is tffr, then $A$ is qElc if and only if $\mathbb{Q} A$ is a cyclic $\mathbb{Q} E n d(A)$-module.

(4) Any direct sum of qElc groups is again qElc.

(5) If $A$ is tffr and qElc, then $\operatorname{rank}(\operatorname{End}(A)) \geq \operatorname{rank}(A)$.

Proof. We will prove (2) first. Assume (2.1) holds. Let $a_{i} \in A$ for all $1 \leq i \leq n$. By the induction hypothesis, there is some $c_{1} \in A$ such that $a_{i} \in(\mathbb{Q} \operatorname{End}(A)) \cdot c_{1}$ for all $1 \leq i \leq n-1$. Since $A$ is qElc, there is some $c_{2} \in A$ such that $c_{1}, a_{n} \in$ $(\mathbb{Q} \operatorname{End}(A)) \cdot c_{2}$. This shows that $a_{i} \in(\mathbb{Q} \operatorname{End}(A)) \cdot c_{2}$ for all $1 \leq i \leq n$, i.e. $(2.2)$ holds. To prove that $(2.2)$ implies $(2.3)$, assume that $a, b \in(\mathbb{Q} E n d(A)) \cdot c$. Then there exist rational numbers $q_{i}=\frac{z_{i}}{n_{i}}, i=1,2$, such that, for some $\alpha, \beta \in \operatorname{End}(A)$, we have that $a=\left(q_{1} \alpha\right)(c)$ and $b=\left(q_{2} \beta\right)(c)$. Note that $n_{1} n_{2} a=\left(z_{1} n_{2} \alpha\right)(c)$ and $n_{1} n_{2} b=\left(z_{2} n_{1} \beta\right)(c)$. We infer that $(2.3)$ holds because $z_{1} n_{2} \alpha, z_{2} n_{1} \beta \in \operatorname{End}(A)$. It is trivial that (2.3) implies (2.1). This finishes the proof of (2). To show (1), we may assume that there is some natural number $n$ such that $n A \subseteq B \subseteq A$. This implies that $\mathbb{Q} A=\mathbb{Q} B$ as well as $\mathbb{Q} \operatorname{End}(A)=\mathbb{Q} \operatorname{End}(B)$. Thus $\mathbb{Q} \mathbb{A}$ is a locally cyclic $\mathbb{Q} E n d(A)$-module if and only if $\mathbb{Q B}$ is a locally cyclic $\mathbb{Q} E n d(B)$-module. Item (3) follows immediately from (2), since $\mathbb{Q} A$ is a finite-dimensional $\mathbb{Q}$-vector space. (4) follows easily from the definitions just like the corresponding result for E-lc groups in [6]. To show (5), assume that $A$ is tffr and qElc. By (3), there is some $c \in A$ such that $\mathbb{Q} A=\mathbb{Q} \operatorname{End}(A) \cdot c$ is an epimorphic image of $\mathbb{Q} \operatorname{End}(A)$ and thus $\operatorname{rank}(\operatorname{End}(A)) \geq \operatorname{rank}(A)$. 
Now we are ready to show that $\operatorname{End}(A)$ is a maximal subring of $M_{0}(A)$ for qElc groups $A$.

Theorem 1. Let $A$ be a torsion-free group such that $A$ is qElc. Then End $(A)$ is a maximal subring of $M_{0}(A)$.

Proof. Let $R$ be a subring of $M_{0}(A)$ containing $\operatorname{End}(A)$. From $[6$ we know that $R \subseteq M_{\mathbb{Z}}(A)=\left\{f \in M_{0}(A): f(n a)=n f(a)\right.$ for all $\left.n \in \mathbb{Z}, a \in A\right\}$. To show that $R=\operatorname{End}(A)$, let $\rho \in R$ and $a, b \in A$. By Lemma $1(2.3)$ there exist $c \in A, 0 \neq n \in \mathbb{Z}$, $\alpha, \beta \in \operatorname{End}(A)$ such that $\alpha(c)=n a$ and $\beta(c)=n b$. Since $\alpha, \beta \in \operatorname{End}(A) \subseteq R$, we have that $\rho \circ(\alpha+\beta)=\rho \circ \alpha+\rho \circ \beta$. We infer

$$
\begin{aligned}
n \rho(a+b) & =\rho(n a+n b)=\rho(\alpha(c)+\beta(c))=(\rho \circ(\alpha+\beta))(c) \\
& =(\rho \circ \alpha+\rho \circ \beta)(c)=\rho(\alpha(c))+\rho(\beta(c))=\rho(n a)+\rho(n b)=n(\rho(a)+\rho(b)) .
\end{aligned}
$$

Since $A$ is torsion-free, we may cancel $n$ and we infer $\rho(a+b)=\rho(a)+\rho(b)$, which shows that $\rho \in \operatorname{End}(A)$ and thus $R=\operatorname{End}(A)$.

While $\operatorname{End}(A)$ is a maximal subring for any torsion group $A$ (cf. [6]), this is no longer true for torsion-free groups $A$ of rank at least 2. Note that subgroups of $\mathbb{Q}$ are actually E-lc since $\mathbb{Q}$ is a locally cyclic group.

Theorem 2. Let $A$ be a torsion-free group of rank at least 2. If $\operatorname{End}(A)$ is (isomorphic to) a subring of $\mathbb{Q}$, then $\operatorname{End}(A)$ is not a maximal subring of $M_{0}(A)$ and $A$ is not qElc.

Proof. For any $0 \neq a \in A$, let $\langle a\rangle_{*}$ denote the pure subgroup of $A$ generated by $a$ and $R=\left\{f \in M_{\mathbb{Z}}(A): f\left(\langle a\rangle_{*}\right) \subseteq\langle a\rangle_{*}\right.$ for all $\left.a \in A\right\}$. It is clear that $R$ is closed under addition and composition, and the identity is in $R$. Thus $R$ is a subnear-ring of $M_{\mathbb{Z}}(A)$. To show that $R$ is also a ring, let $\alpha, \beta, \gamma \in R$ and $a \in A$. Then there exist $r, s \in \mathbb{Q}$ such that $\beta(a)=r a$ and $\gamma(a)=s a$. Since any integral multiple of an element in $R$ is also in $R$, we may take $r, s$ to be integers. Then

$$
\begin{aligned}
& (\alpha \circ(\beta+\gamma))(a)=\alpha(\beta(a)+\gamma(a))=\alpha((r+s) a)=(r+s) \alpha(a) \\
& \quad=r \alpha(a)+s \alpha(a)=\alpha(r a)+\alpha(s a)=\alpha(\beta(a))+\alpha(\gamma(a))=(\alpha \circ \beta+\alpha \circ \gamma)(a),
\end{aligned}
$$

which shows that $R$ is left distributive and therefore a ring. Since the rank of $A$ is at least 2 , it is not hard to show that $R \neq \operatorname{End}(A)$. Clearly, $\operatorname{End}(A) \subseteq \mathbb{Q}$ is also contained in $R$. This shows that $\operatorname{End}(A)$ is not a maximal subring of $M_{0}(A)$.

We remark that the absolutely anisotropic groups of rank at least 2 in [4] and [5] are examples of groups satisfying the conditions of Theorem 2. In this case, the ring $R$ in the proof of Theorem 2 is $M_{\mathbb{Z}}(A)$ and $\operatorname{End}(A) \varsubsetneqq M_{\mathbb{Z}}(A)$.

We also note that, from the above theorem, that if $A$ is qElc, then $\operatorname{End}(A) \varsubsetneqq \mathbb{Q}$. In Example 3 we will give an example of a group $A$ with $\operatorname{End}(A) \varsubsetneqq \mathbb{Q}$ such that $A$ is not qElc. Thus the converse of Theorem 2 does not hold.

We will now present an example of a tffr group $G$ such that $G$ is qElc, but not E-lc.

Example 1 (see [2, page 158]). Let $p, p_{1}, p_{2}, p_{3}$ be distinct prime integers, and let $X_{i}$ be the subring of $\mathbb{Q}$ generated by $\frac{1}{p_{i}}, i=1,2,3$. Let $X=\bigoplus_{i=1}^{3} X_{i}$ and let $G=X+\frac{1}{p}(1,0,1) \mathbb{Z}+\frac{1}{p}(0,1,1) \mathbb{Z}$. Then $G \cap \mathbb{Q} X_{i}=X_{i}$ and, using types, one finds that each $X_{i}$, hence $X$, is fully invariant in $G$. Further, $G / X$ is a 2-dimensional vector space over $\mathbb{Z}_{p}$, the field with $p$ elements. By way of contradiction, assume 
that $G$ is E-lc. Let $\left\{b_{1}+X, b_{2}+X\right\}$ be a basis of the $\mathbb{Z}_{p}$-vector space $G / X$. Then there exist $c \in G, \varphi_{1}, \varphi_{2} \in \operatorname{End}(G)$ such that $\varphi_{i}(c)=b_{i}, i=1,2$. Since $X$ is fully invariant in $G$, the $\varphi_{i}$ induce endomorphisms $\psi_{i}$ of $G / X$ such that $\psi_{i}(c+X)=b_{i}+X$, and we have that $G / X$ is a cyclic $\operatorname{End}(G)$-module. This is a contradiction, since some routine computations show that $\operatorname{End}(G)$ induces only scalar multiplications on $G / X$. Thus $G$ is not E-lc. On the other hand, $G$ is quasiequal to $X$, which is, by Lemma $1(4)$, a qElc group. Therefore, by Lemma 1 (4), we have that $G$ is qElc.

Using any group $A$ from Example 1, we have that $\operatorname{End}(A)$ is a maximal subring of $M_{0}(A)$, but $A$ is not E-lc. This shows that the converse of [6, Theorem I.2] does not hold. It is still open if the converse of our Theorem 1 holds.

\section{RANK 2 GROUPS}

In this section we will use the results above and Arnold's work 1 to completely determine which torsion-free groups of rank 2 are qElc. Such a group $G$ is either almost completely decomposable, i.e. $G$ is quasi-equal to a completely decomposable group, or strongly indecomposable, i.e. $\mathbb{Q} E n d(G)$ has only the trivial idempotents. Recall that any rank 1 group $A \subseteq \mathbb{Q}$ is locally cyclic and thus $A$ is E-lc and qElc. By Lemma 1(4) we have that completely decomposable groups are qElc and Lemma 1(1) implies that any almost completely decomposable group is qElc. Therefore, we may now assume that $G$ is strongly indecomposable. Arnold [1. Theorem 3.3] states that these groups $G$ fall into four distinct classes according to the size of the type set of $G$. For two of these classes one has that $\operatorname{End}(G)$ is a subring of $\mathbb{Q}$ and $G$ is not qElc by Theorem 2. A third class of rank-2 groups $G$ has $\mathbb{Q} \operatorname{End}(G) \approx\left\{\left[\begin{array}{ll}x & 0 \\ y & x\end{array}\right]: x, y \in \mathbb{Q}\right\}$ and we see that the 2-dimensional $\mathbb{Q}$ vectorspace $\mathbb{Q} G$ is a cyclic $\mathbb{Q} E n d(G)$-module with generator $\left[\begin{array}{l}1 \\ 1\end{array}\right]$ and thus qElc by Lemma $1(3)$. In the fourth and last case, $\mathbb{Q} \operatorname{End}(G)$ is a quadratic number field, i.e. a field $F$ such that $\operatorname{dim}_{\mathbb{Q}}(F) \leq 2$. Of course, if $\operatorname{dim}_{\mathbb{Q}}(F)=1$, then $\operatorname{End}(G)$ is a subring of $\mathbb{Q}$ and thus $G$ is not qElc, as seen above. On the other hand, if $\operatorname{dim}_{Q}(F)=2$, then $\mathbb{Q} G$ is a cyclic $\mathbb{Q} E n d(G)$-module and thus $G$ is qElc. We conclude that either $G$ is qElc or $\operatorname{End}(G)$ is a subring of $\mathbb{Q}$. Theorem 2 tells us that in the latter case, $\operatorname{End}(G)$ is not a maximal subring of $M_{0}(G)$.

We summarize the above discussion in the following theorem.

Theorem 3. Let $G$ be a torsion-free group of rank 2. Then the following are equivalent:

(1) $G$ is $q E l c$.

(2) $\mathbb{Q} \operatorname{End}(G)$ is not isomorphic to $\mathbb{Q}$.

(3) $\operatorname{dim}_{\mathbb{Q}}(\mathbb{Q} \operatorname{End}(G)) \geq \operatorname{rank}(G)=2$.

(4) $\operatorname{End}(G)$ is a maximal subring of $M_{0}(G)$.

When $G$ is strongly indecomposable, then the inequality in (3) becomes an equality.

Corollary 1. Let $G$ be a torsion-free group of rank 2. Then End $(G)$ is a maximal subring of $M_{0}(G)$ if and only if $\operatorname{dim}_{\mathbb{Q}}(\mathbb{Q} E n d(G))>1$. 


\section{General Results}

In this section we let $A$ be any torsion-free group of finite rank (tffr) and determine some general conditions under which $A$ is qElc. Recall [1, Corollary 7.9] that $A$ is quasi-isomorphic to a direct sum of strongly indecomposable groups.

Theorem 4. Let $A$ be a tffr group. If $A$ is quasi-isomorphic to a (finite) direct sum of strongly indecomposable tffr qElc groups, then $A$ is qElc. The converse of this statement does not hold.

Proof. If $A$ is quasi-isomorphic to a direct sum of qElc groups, then $A$ is qElc by Lemma 1(4) and (1). On the other hand, pick a tffr group $B$ of rank at least 2 such that $\mathbb{Q} \operatorname{End}(B)=\mathbb{Q}$. Then $B$ is not qElc by Theorem 2. Let $A=\mathbb{Z} \oplus B$. Then $A$ is E-lc by [6, Theorem III.3]. This shows that the class of qElc groups is not closed under (quasi-) summands.

We now focus our attention on strongly indecomposable groups. The next result can be found in [1].

Theorem 5 (11, Corollary 7.8]). If $A$ is a tffr group, then $A$ is strongly indecomposable if and only if $\mathbb{Q} E n d(A)$ is a local ring.

Now from Wedderburn's Principal Theorem, $\mathbb{Q} \operatorname{End}(A)=D+J(\mathbb{Q} \operatorname{End}(A))$, where $D$ is a division ring and the Jacobson radical $J=J(\mathbb{Q} E n d(A))$ is nilpotent. Suppose $J(\mathbb{Q} E n d(A))=\{0\}$. If $A$ is qElc, we know from Lemma $1(3)$ that $\mathbb{Q} A$ is a cyclic $\mathbb{Q} \operatorname{End}(A)$-module. Hence there is some $c \in A$ such that $\mathbb{Q} A=D c$. Since $D$ is a division ring, we get that $\operatorname{dim}_{\mathbb{Q}}(D)=\operatorname{rank}(A)$. We include this in our next result.

Theorem 6. Let $A$ be a strongly indecomposable tffr group such that $\mathbb{Q} E n d(A)=D$ is a division ring, i.e. $J=\{0\}$. Then the following are equivalent:

(1) $A$ is qElc.

(2) $\operatorname{rank}(\operatorname{End}(A))=\operatorname{dim}_{\mathbb{Q}}(D)=\operatorname{dim}_{\mathbb{Q}}(\mathbb{Q} A)=\operatorname{rank}(A)$.

(3) $\operatorname{dim}_{D}(\mathbb{Q} A)=1$.

(4) $D x=D y$ for all $0 \neq x, y \in \mathbb{Q} A$.

(5) $D x \cap D y \neq\{0\}$ for all $0 \neq x, y \in \mathbb{Q} A$.

Proof. In the remarks prior to the theorem we have established that (1) implies (2). Since $\operatorname{dim}_{\mathbb{Q}}(D)=\operatorname{dim}_{\mathbb{Q}}(\mathbb{Q} A), D$ and $\mathbb{Q} A$ are isomorphic $\mathbb{Q}$-spaces and since both are $D$-spaces, we get that $\operatorname{dim}_{D}(\mathbb{Q} A)=1$. In this case, for any $0 \neq x, y \in \mathbb{Q} A$, $D x=D y=\mathbb{Q} A$, so we have that (3) implies (4). Trivially, (4) implies (5), and it remains to be shown that (5) implies (1). To this end, let $x, y$ be any non-zero elements of $\mathbb{Q} A$ and let $0 \neq c \in D x \cap D y$. Thus there exist $d_{1}, d_{2} \in D$ with $c=d_{1} x=d_{2} y$. Since $D$ is a division ring, $x=d_{1}^{-1} c$ and $y=d_{2}^{-1} c$, which shows that $A$ is qElc.

In particular, under the above hypothesis, $A$ is qElc if and only if $\operatorname{rank}(\operatorname{End}(A))$ $=\operatorname{rank}(A)$. The following example shows that this is not true in general.

Example 2. Let $A$ be tffr of rank $n$ with $\mathbb{Q} E n d(A)=\mathbb{Q}$ (see [3, page 128, Exercise 8]). Let $m$ be an integer with $m>n$ and $B=A^{m}$. Then $\mathbb{Q} E n d(B) \approx \operatorname{Mat}_{m \times m}(\mathbb{Q})$, the ring of all $m \times m$-matrices over $\mathbb{Q}$. Let $\left\{a_{1}, \ldots, a_{n}\right\} \subset A$ be a basis of $\mathbb{Q} A$ over $\mathbb{Q}$. Then the $m$-tuple $\left(a_{1}, a_{2}, \ldots, a_{n}, 0, \ldots, 0\right) \in B$ generates $\mathbb{Q} B$ as a $\mathbb{Q} E n d(B)$-module. Therefore $B$ is qElc, but $\operatorname{rank}(B)=m n<m^{2}=\operatorname{rank}(\operatorname{End}(B))$. 
We next present some additional conditions which allow us to conclude that a strongly indecomposable torsion-free group is qElc. From the proof of Theorem 6 we note that if $D x \cap D y \neq\{0\}$ for all $0 \neq x, y \in \mathbb{Q} A$, then independent of $J=\{0\}$, we get that $A$ is qElc. Thus we wish to determine conditions under which $D x \cap D y \neq\{0\}$. We know $\operatorname{dim}_{\mathbb{Q}}(D x)=\operatorname{dim}_{\mathbb{Q}}(D y)=\operatorname{dim}_{\mathbb{Q}}(D)$. Moreover, $\operatorname{dim}_{\mathbb{Q}}(D x+D y) \leq \operatorname{dim}_{\mathbb{Q}}(\mathbb{Q} A)=n$. Thus $n \geq \operatorname{dim}_{\mathbb{Q}}(D x+D y)=\operatorname{dim}_{\mathbb{Q}}(D x)+$ $\operatorname{dim}_{\mathbb{Q}}(D y)-\operatorname{dim}_{\mathbb{Q}}(D x \cap D y)=2 \operatorname{dim}_{\mathbb{Q}}(D)-\operatorname{dim}_{\mathbb{Q}}(D x \cap D y)$. Therefore

$$
\operatorname{dim}_{\mathbb{Q}}(D x \cap D y) \geq 2 \operatorname{dim}_{\mathbb{Q}}(D)-n .
$$

Hence if $\operatorname{dim}_{\mathbb{Q}}(D)>\frac{n}{2}$, then we have $\operatorname{dim}_{\mathbb{Q}}(D x \cap D y)>0$.

Theorem 7. Let $A$ be strongly indecomposable with $\mathbb{Q} E n d(A)=D+J$. If $2 \cdot \operatorname{dim}_{Q}(D)>\operatorname{rank}(A)$, then $A$ is $q$ Elc.

We will see in the next theorem that the converse need not be true.

We continue with $A$ strongly indecomposable and $\mathbb{Q} \operatorname{End}(A)=D+J$, with $J \neq\{0\}$. We know that $A$ is qElc if and only if there exists some $c \in \mathbb{Q} A$ such that $\mathbb{Q} A=(\mathbb{Q} \operatorname{End}(A)) \cdot c=D c+J c$. Moreover, $D c \cap J c=\{0\}:$ If $y \in D c \cap J c=J c \cap c D$, then $y=d c=j c$ for some $d \in D$ and $j \in J$. If $y \neq 0$, then $c=d^{-1} j c$ and so $\left(1-d^{-1} j\right) c=0$. But $1-d^{-1} j$ is invertible since $J$ is nilpotent and we get $c=0$, a contradiction. Thus we have that $A$ is qElc if and only if there is some $c \in \mathbb{Q} A$ such that $\operatorname{rank}(A)=\operatorname{dim}_{\mathbb{Q}}(D c)+\operatorname{dim}_{\mathbb{Q}}(J c)$. In particular, suppose we consider, instead of $J$ being as small as possible, that $D$ be as small as possible, i.e. $D=\mathbb{Q}$. We have established the following result.

Theorem 8. Let $A$ be a strongly indecomposable group with $\mathbb{Q} E n d(A)=\mathbb{Q}+$ $J, J \neq\{0\}$. Then $A$ is qElc if and only if there exists some $c \in \mathbb{Q} A$ such that $\operatorname{dim}_{\mathbb{Q}}(J c)=\operatorname{rank}(A)-1$.

It would be interesting to determine when the conditions of the above theorem are satisfied. One situation is the following: Suppose there exist $j_{1}, \ldots, j_{n-1} \in J-\{0\}$ and $c \in \mathbb{Q} A$ such that $j_{1}(c), \ldots, j_{n-1}(c) \in J(c)=J \cdot c$ are $\mathbb{Q}$-linearly independent elements of $J(c)$. Since, as we have seen, $J(c) \neq \mathbb{Q} A$, so we have $\operatorname{dim}_{\mathbb{Q}}(J(c))=n-1$ where $\operatorname{rank}(A)=n$.

Suppose that $\operatorname{rank}(A)=p$ is a prime and again let $A$ be tffr strongly indecomposable with $\mathbb{Q} E n d(A)=D+J$. If $\operatorname{dim}_{\mathbb{Q}}(D)=\ell$ and $\operatorname{dim}_{D}(\mathbb{Q} A)=t$, then $p=\ell t$ and so $\ell=1$ or $\ell=p$. If $\ell=1$ and $J=\{0\}$, then $A$ is not qElc. If $\ell=1$ and $J \neq\{0\}$, then Theorem 8 applies. If $\ell=p$, then $\operatorname{dim}_{D}(\mathbb{Q} A)=1$, so for each $0 \neq c \in \mathbb{Q} A$ we have that $D c=\mathbb{Q} A$. This in turn implies $J=\{0\}$, and Theorem 6 applies. Thus one knows when strongly indecomposable groups of prime rank are qElc.

We conclude with a class of examples which show that the conditions of Theorem 8 may or may not hold.

Example 3. From $[8$, if $R$ is a ring with free additive group of rank $n$, then there exists a torsion-free group $A$ of rank $n$ such that $\operatorname{End}(A)=R$. In fact $A$ is a subgroup of $\mathbb{Q} R$ and strongly indecomposable if $\mathbb{Q} R$ has only the trivial idempotents 0,1 . We take $R$ to be the subring of $M_{n t_{n \times n}}(\mathbb{Z})$ of all lower triangular matrices with the same entry along the main diagonal. Then $R=\mathbb{Z} \oplus N$ with $N$ nilpotent and $\mathbb{Q} \operatorname{End}(A)=\mathbb{Q} R=\mathbb{Q} \oplus \mathbb{Q} N=\mathbb{Q} \oplus J$. For ease of exposition of our examples, we 
take $n=3$. If $R_{1}=\left\{\left[\begin{array}{lll}a & 0 & 0 \\ b & a & 0 \\ c & 0 & a\end{array}\right]: a, b, c \in \mathbb{Z}\right\}$, then $\operatorname{rank}\left(R_{1}\right)=\operatorname{rank}(A)=3$ and $\mathbb{Q} \operatorname{End}(A)=\mathbb{Q} \oplus J$ with $J=\left\{\left[\begin{array}{lll}0 & 0 & 0 \\ x & 0 & 0 \\ y & 0 & 0\end{array}\right]: x, y \in \mathbb{Q}\right\}$. From this we see, for $c=\left[\begin{array}{l}1 \\ 0 \\ 0\end{array}\right]$, that $\operatorname{dim}_{\mathbb{Q}}(J c)=2=\operatorname{rank}(A)-1$ and it follows that $A$ is qElc. On the other hand, if $R_{2}=\left\{\left[\begin{array}{lll}a & 0 & 0 \\ 0 & a & 0 \\ b & c & a\end{array}\right]: a, b, c \in \mathbb{Z}\right\}$, then again $\operatorname{rank}(A)=$ 3 and $\mathbb{Q} \operatorname{End}(A)=\mathbb{Q}+J$ with $J=\left\{\left[\begin{array}{lll}0 & 0 & 0 \\ 0 & 0 & 0 \\ x & y & 0\end{array}\right]: x, y \in \mathbb{Q}\right\}$. Here, $\operatorname{dim}_{\mathbb{Q}}(J c)=$ $1 \neq \operatorname{rank}(A)-1$ for any $c \in \mathbb{Q}^{3}$, so $A$ is not qElc.

\section{REFERENCES}

[1] Arnold, D. M., Finite Rank Torsion-Free Abelian Groups and Rings, LNM 931, SpringerVerlag, New York, 1982. MR0665251 (84d:20002)

[2] Arnold, D. M., Abelian Groups and Representations of Finite Partially Ordered Sets, CMS Books in Mathematics, Springer-Verlag, New York, 2000. MR1764257 (2001g:16030)

[3] Fuchs, L., Infinite Abelian Groups, vols. I and II, Academic Press, New York, 1970 and 1973. MR0255673 (41:333) MR0349869 (50:2362)

[4] Hausen, J., Abelian groups whose semi-endomorphisms form a ring, Abelian Groups, Marcel Dekker, New York, 1993, pp. 175-180. MR1217268 (94c:20096)

[5] Hausen J. and Johnson, J., Centralizer near-rings that are rings, J. Austral. Math. Soc. (Series A) 59, (1995), 173-183. MR.1346625 (96g:16061)

[6] Kreuzer, A. and Maxson, C. J., E-locally cyclic abelian groups and maximal near-rings of mappings, Forum Mathematicum, to appear.

[7] Pilz, G. F., Near-Rings, 2nd ed. North-Holland, 1963. MR0469981 (57:9761)

[8] Zassenhaus, H., Orders as endomorphism rings of modules of the same rank, J. London Math. Soc. 42, (1967), 180-182. MR0206051(34:5876)

Department of Mathematics, Baylor University, Waco, Texas 76798

E-mail address: Manfred_Dugas@baylor.edu

Department of Mathematics, Texas A\&M University, College Station, Texas 77843

E-mail address: cjmaxson@math.tamu.edu 